\section{Breaking the Resolution Barrier in the Scanning Electron Microscope}

\author{
William Vanderlinde \\ Laboratory for Physical Sciences \\ College Park, Maryland, USA \\ billv@lps.umd.edu
}

\section{Introduction}

Everyone always wants better resolution from his or her microscopes. With semiconductor manufacturers now shipping product with sub-100 nm gates, measuring features and defects has become a challenge, even for the scanning electron microscope (SEM). For metrology below $100 \mathrm{~nm}$, some manufacturers have begun routinely using TEM (transmission electron microscopy) which is tedious and expensive. [1] As a microscopist, I find this quite disappointing since, in principle, the SEM should be capable of providing more than enough resolution well below $100 \mathrm{~nm}$. Why is it that SEMs with $1 \mathrm{~nm}$ spot size can't provide adequate resolution for $100 \mathrm{~nm}$ gates? It turns out that at very high magnification, SEM resolution is limited by how the electron beam interacts with the sample rather than simply the spot size of the beam.

In this article I will describe two innovative methods for significantly improving resolution in SEM imaging: STEM-in-SEM (scanning transmission electron microscopy in a scanning electron microscope) $[2,3,4]$, and FSEI (forward scattered electron imaging). [5] Both methods can be implemented in any SEM by using special sample holders and do not require any modification to the SEM.

\section{Resolution Barriers in the SEM}

Modern field emission SEMs have a nominal spot size as small as $1 \mathrm{~nm}$. However, it is very rare that $1 \mathrm{~nm}$ resolution is realized in everyday imaging. Typically, a SEM service engineer will demonstrate the microscope resolution by using very high beam voltage such as $30 \mathrm{keV}$ to image a special "resolution standard" that consists of gold islands on a carbon substrate-commonly referred to as a "gold-on-carbon" sample-see Figure 1, taken at extremely high $500,000 x$ magnification. Imaging a more typical sample such as an electronic device will generally produce an image with much lower resolution that may not meet the needs of modern failure analysis and metrology.

It is sometimes possible to achieve very high resolution by sputter coating a very thin layer of metal, such as $\mathrm{Au}, \mathrm{Au} / \mathrm{Pd}, \mathrm{Cr}$, or Pt on the sample surface. Typically, these coatings result in a better image of the sample surface, because they eliminate charging, increase the surface emissivity of the sample, and reduce the secondary electron
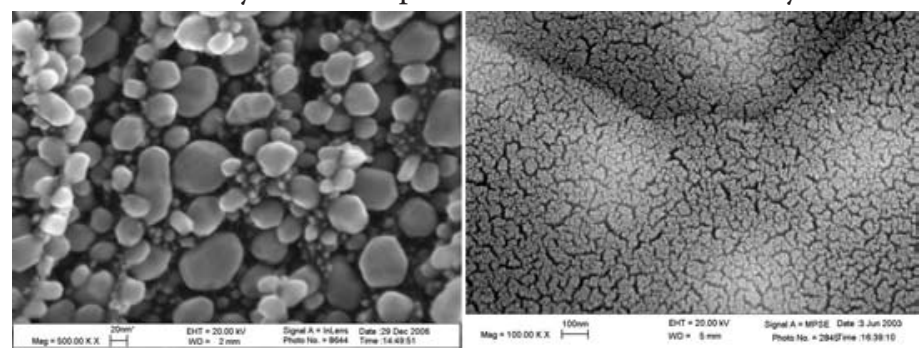

Figure 1, left: Secondary electron image of gold islands on a carbon substrate shows that very high resolution SEM images are possible under the right conditions.

Figure 2, right: Secondary electron image of aluminum metal sputter coated with $10 \mathrm{~nm}$ of $\mathrm{Au} / \mathrm{Pt}$. The image has high resolution but the original surface detail is obscured and the sputter coating grains introduce artifacts seen as jagged dark lines. range. About $10 \mathrm{~nm}$ of metal is required to produce a sputtered film, but this amount of metal may obscure surface detail and cause image artifacts from the grain structure of the sputtered metal. It is therefore unacceptable to sputter coat for imaging at 1 nm resolution (see Figure 2).

In order to achieve ultra-high resolution in the SEM, three conditions must be satisfied: [6]

1. The electron beam must be finely focused to a small spot at the surface of the sample.

2. The electron beam current must be high enough to produce sufficient signal so that visible contrast is achieved in the image.

3. The signal used for imaging must originate very close to the impact area of the beam on the sample.

Conditions 1 and 2 are satisfied when the electron source is operating at high brightness, which is maximized when the electron source is at high beam voltage. However, high energy electrons have a large range in most materials. When the electrons are backscattered from deep in the sample back towards the surface, they may exit the sample at a significant distance from the probe location (see Table 1.) Thus, condition 3 is not satisfied at high voltage since the backscattered electrons are not closely correlated with the probe. These electrons also produce secondary electrons as they exit and this further reduces the spatial correlation between the secondary electrons and the probe. If the beam voltage is reduced so as to decrease the range of the electrons, then the brightness of the electron source is reduced and it is not possible to achieve a small spot with sufficient beam current (conditions 1 and 2). For the special gold-on-carbon sample, all three conditions are satisfied at high beam voltage because gold has a very high backscatter coefficient and carbon has a very low backscatter coefficient. Thus, incident electrons are usually either reflected from the gold surface or lost in the carbon substrate. However, it is very difficult to find ordinary samples that meet all three conditions.

Table 1: Electron beam spot size (per the manufacturer [7]) and penetration range (from the Kanaya-Okayama formula [8]) as a function of electron beam energy.

\begin{tabular}{|c|c|c|}
\hline $\begin{array}{c}\text { Beam energy } \\
(\mathrm{keV})\end{array}$ & Spot size $(\mathrm{nm})$ & $\begin{array}{c}\text { Electron penetration } \\
\text { range in aluminum } \\
\text { (microns) }\end{array}$ \\
\hline 1 & 2.4 & 0.028 \\
\hline 3.5 & 1.5 & 0.22 \\
\hline 5 & 1.3 & 0.41 \\
\hline 10 & 1.1 & 1.32 \\
\hline 20 & 1.0 & 4.19 \\
\hline 30 & 1.0 & 8.24 \\
\hline
\end{tabular}

\section{STEM-in-SEM}

One solution to satisfying the three conditions for highresolution imaging is to create a very thin sample ( $\leq 100 \mathrm{~nm}$ thick) and image using the transmitted high-energy electrons. Electrons will scatter very little as they pass through a thin film. Therefore, the detected signal is highly localized, even at high beam voltage where the electron optics produce the best spot size. This approach produces high-resolution STEM images that look like TEM images. FIB techniques allow the creation of "TEM samples" less than 100 


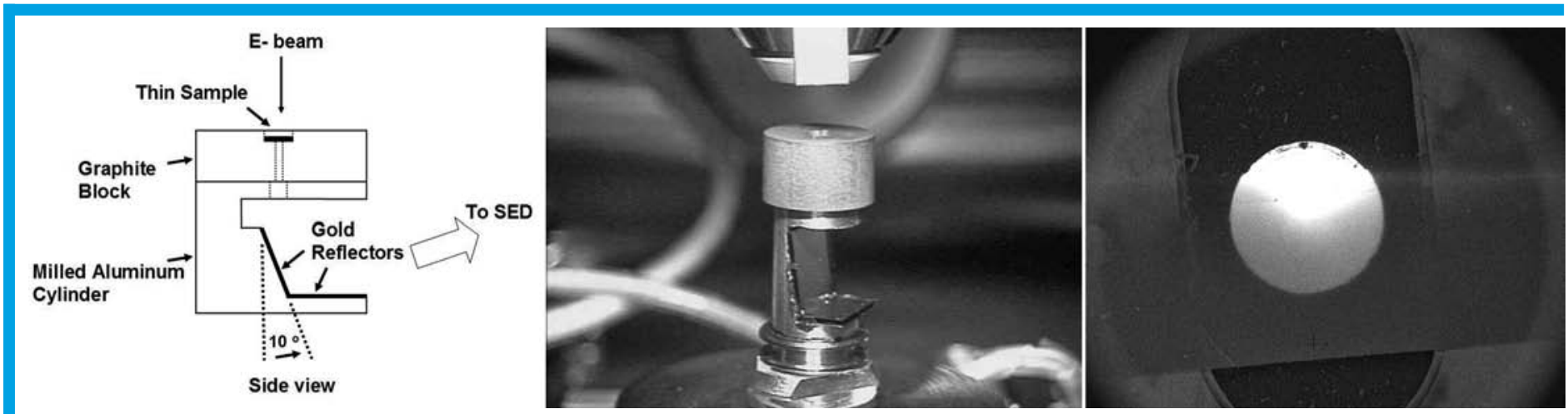

Figure 3, left: Design for a "STEM-in-SEM" sample holder. The overall height is $2.5 \mathrm{~cm}$.

Figure 4: Optical photo of the STEM-in-SEM sample holder mounted to the SEM stage. The pole piece of the objective lens is visible above the sample holder.

Figure 5, right: Low magnification STEM-in-SEM image showing the TEM sample.

$\mathrm{nm}$ thick much easier and faster than in years past.

A home-built "STEM-in-SEM" holder is shown in schematic in Figure 3 and in an optical photo in Figure 4. [2] The thin sample is mounted on top of a graphite block. After passing through the thin film, the electron beam travels down a narrow hole drilled in the graphite block. By absorbing the most highly scattered electrons, the graphite block collimates the beam, which improves the image resolution. After passing through the collimator, the electrons strike a pair of gold reflectors that create secondary electrons that are collected by the ordinary in-chamber secondary electron detector. A similar STEM-in-SEM holder with a slit-type collimator can be purchased from Ernest Fullam, Inc. as Item No. 18300. Alternately, one can invest in a dedicated STEM-in-SEM detector which can be added to some SEM models. [4]

STEM-in-SEM samples are typically mounted onto a standard $3 \mathrm{~mm}$ diameter TEM disk, which is then placed into a cylindrical hole in the top of the STEM-in-SEM holder and held in place by a retaining ring. However, the STEM-in-SEM holder can be easily modified for a variety of sample shapes and lengths. When thin samples are prepared by FIB, the $3 \mathrm{~mm}$ limitation on sample size imposed by standard TEMs can significantly increase the difficulty of the preparation. With STEM-in-SEM, the sample can be of virtually any size. It is, however, crucial that the sample be mounted in a clean and mechanically rigid fashion. Metallic tape is often used to mount SEM samples, but tape will allow unacceptable sample drift. Carbon paint is also used to mount SEM samples, but with STEM-in-SEM the paint may transfer carbon contamination to the thin section. Therefore, a mechanical mounting method such as spring clips or retaining rings is recommended.

Transmission electron microscopes typically operate at 100 $300 \mathrm{keV}$ or higher, so it may be somewhat surprising that an SEM's electron beam voltage of $30 \mathrm{keV}$ or less can be used to view a sample in transmission. While it is often said that TEM samples must be thinned until they are "electron transparent," this is a misleading statement. The electron range of a $30 \mathrm{keV}$ electron in aluminum is more than 8 microns. TEM samples are typically thinned to less than $100 \mathrm{~nm}$ thick so that most of the electrons undergo no more than one scattering event. Accordingly, TEM imaging is typically done with electrons that have not undergone any scattering events (bright field) or those that have been scattered exactly once by the lattice (dark field). At $200 \mathrm{keV}$ electrons will usually pass through the sample being scattered at most once, but $30 \mathrm{keV}$ electrons will scatter many times. This is why high voltage is generally required for transmission electron microscopy. To improve resolution at 30
$\mathrm{keV}$, my graphite collimator [2] in the STEM-in-SEM holder below the sample, absorbs the most highly scattered electrons that would normally degrade the image-resulting in a high-resolution STEM image in a conventional SEM.

\section{Procedure}

Figure 5 is a low magnification STEM-in-SEM image. The image shows a conventional TEM sample, consisting of a thinned strip of silicon attached to a $3 \mathrm{~mm}$ copper grid with an oval hole. The bright circle in the center of the picture corresponds to the hole in the graphite collimator that allows electrons to penetrate to the gold reflectors. If necessary, the holder can be tilted to bring the area of interest to the center of the bright circle, and the entire holder is then rotated to maximize the collection of secondary electrons from the gold reflectors to the secondary electron detector. Once the holder is aligned, high-resolution STEM-in-SEM images can be acquired by selecting a thin area of the sample and simply increasing the magnification, and then focusing and stigmating in the usual fashion.

\section{Semiconductor cross-sections}

Figures 6, 7, 8, and 9, are STEM-in-SEM images of typical CMOS structures such as aluminum lines, tungsten plugs, and poly lines with sidewall spacers. These images were taken with $20 \mathrm{keV}$ or $30 \mathrm{keV}$ beam voltage and $2 \mathrm{~mm}$ working distance. The STEM-in-SEM images have a similar appearance and contrast compared to TEM images. They show internal grain structures on the poly-silicon, the aluminum, and the barrier metals. Close examination of the STEM-in-SEM images demonstrates a resolution of approximately $2 \mathrm{~nm}$ which is far superior to that achieved by traditional SEM images of these structures. The STEM-in-SEM image contrast is based on differences in atomic number, density, and crystallographic orientation, all of which influence how electrons scatter away from the detector. [9]

STEM-in-SEM also allows for very high spatial resolution energy dispersive $\mathrm{X}$-ray elemental maps. X-rays maps with $10 \mathrm{~nm}$ spatial resolution have been demonstrated. [2,3] However, STEMin-SEM requires the preparation of thin sample. Ideally, one would like a SEM technique that provides very high resolution without requiring any special sample preparation. One such technique is discussed in the next section of this article.

\section{Forward Scattered Electron Imaging}

A second method for satisfying the three conditions for ultrahigh resolution SEM is to image using low-loss electrons. This is actually a very old idea that can be found in literature dating back to 


\section{SII

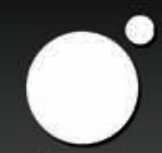

SII NanoTechnology USA Inc.

\section{Silicon Drift Detector}

NO LN2 • Active area $50 \mathrm{~mm}^{2} \bullet<130 \mathrm{eV}$ FWHM at $5.9 \mathrm{keV} \bullet$ ICR $1.5 \mathrm{Mcps} \bullet 0$ CR up to $600 \mathrm{kcps}$

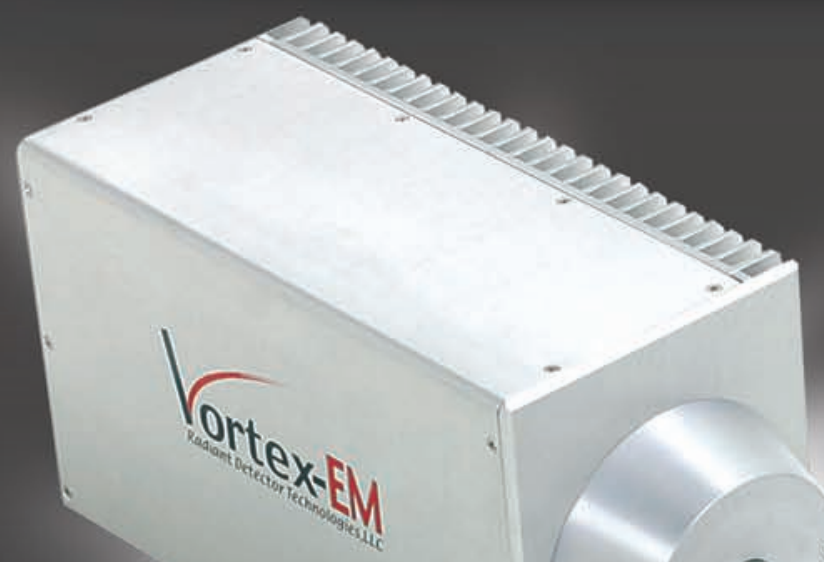

\section{SO FAST}

IT WILL TAKE

YOUR "BREAK" AWAY!

$\mathrm{C}$ and the $\mathrm{B}$ peaks are completely separated from noise by the Vortex-EM detector. It therefore, facilitates light element detection while performing microanalysis and fast elemental mapping applications.

\section{BREAKTHROUGH PERFORMANCE}

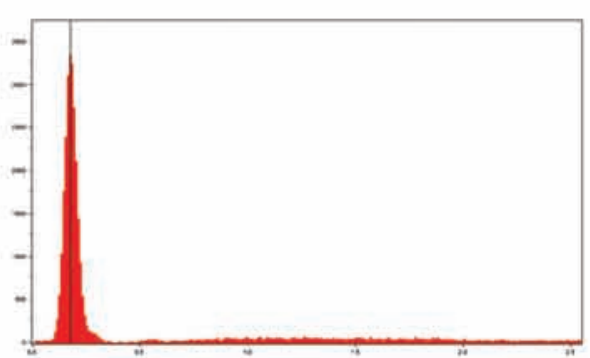

Boron

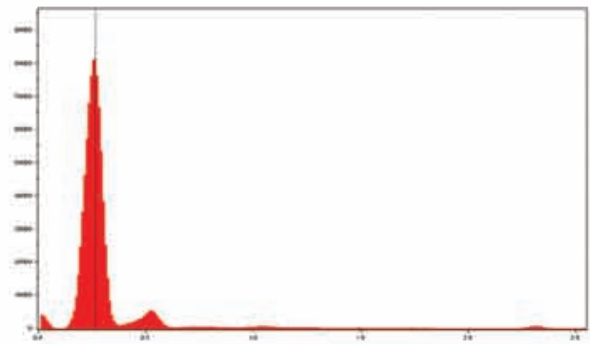

Carbon

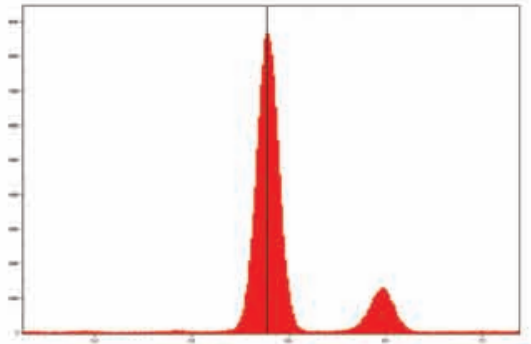

$129 \mathrm{eV}$ Mn sample spectrum

EDS • Fast Mapping $\odot$ SEM $・$ Microanalysis $・$ Process Control $\bullet$ Synchroton $\circ$ TXRF $\circ$ SR-TXRF 


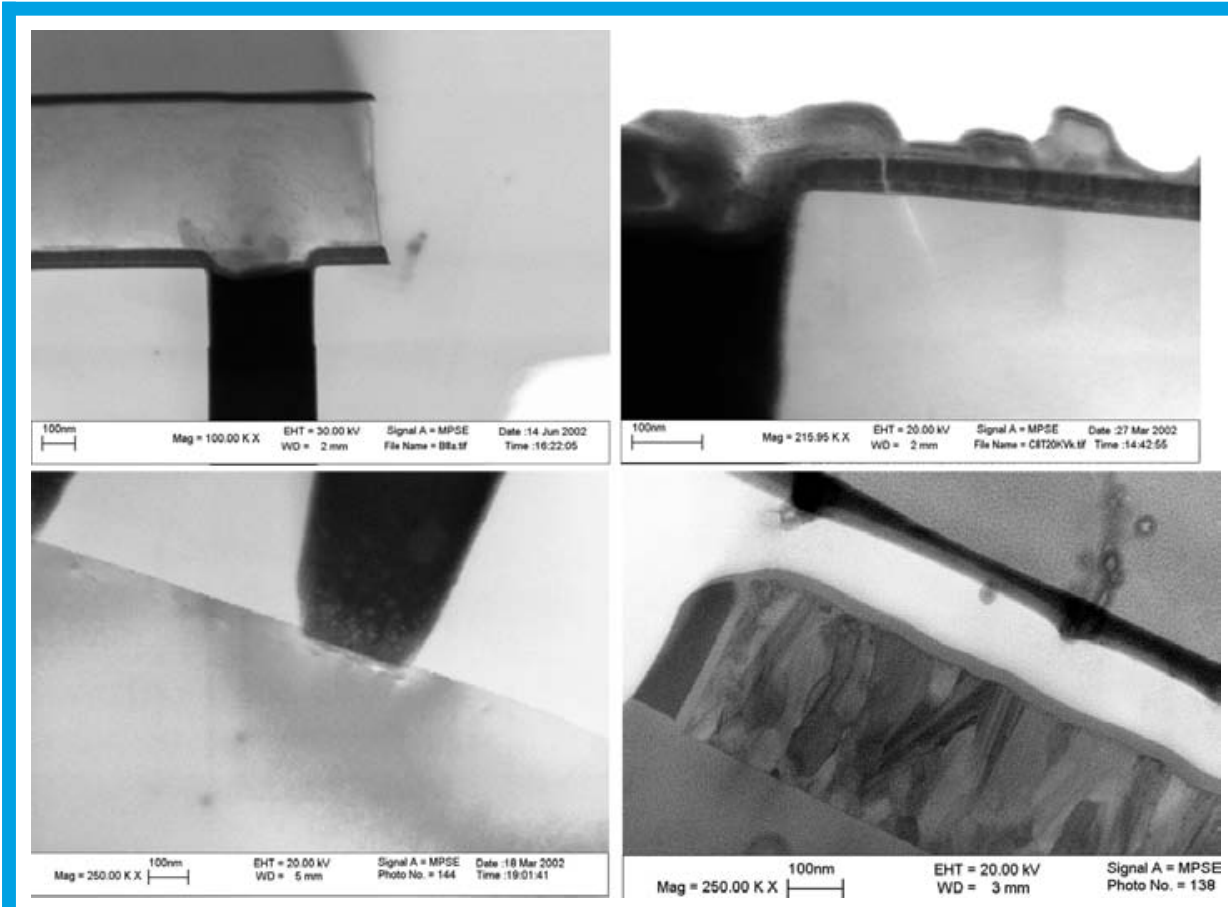

Figure 6, top left: STEM-in-SEM image of an aluminum line connected to a tungsten plug contact. The barrier layer and the plug liner are visible.

Figure 7, top right: Higher magnification STEM-in-SEM image resolving a titanium plug liner and barrier layer.

Figure 8, bottom left: STEM-in-SEM image of a tungsten plug contacting silicon.

Figure 9, bottom right: STEM-in-SEM of broad poly-silicon line with sidewall spacer.

the 1950s. Unfortunately, it used to be rather difficult to implement low-loss imaging because it required major changes to the SEM chamber. Recently I invented a special sample holder that provides images that are very similar to low-loss images.

The basic idea in low-loss imaging is that electrons with the smallest energy loss will have experienced the smallest path length through the material and thus will be highly localized to the beam impact area. When the beam is at normal incidence, very few electrons escape the sample with low loss. However, when an electron beam enters a sample at very high tilt angle compared to the sample normal, a significant fraction of the incident electrons will be forward scattered from the sample surface with relatively little energy loss. The low-loss signal is typically detected by a large acceptance angle ( $~ 45$ degrees) energy-filtered detector placed below the sample, as developed by Wells at IBM. $[10,11,12]$ However, this requires the expense and complication of adding a special detector to the microscope chamber.

Interestingly, some very high spatial resolution "low-loss" images were obtained by Broers using a very different set-up, [13]

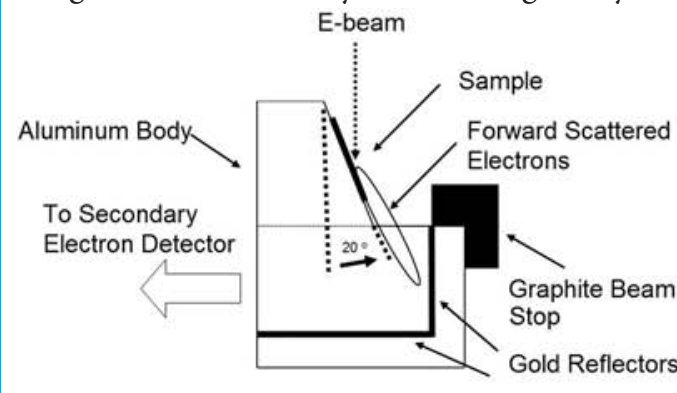

Sample Holder - Side View
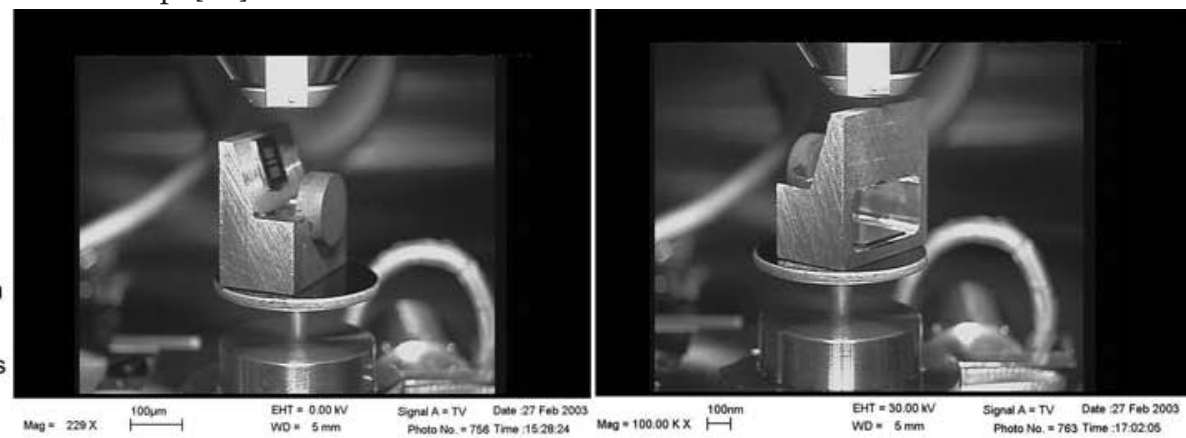
Signal A=TV Dete 27 Fab 2000

Figure 10, left: Design of a sample holder with an integrated detector for forward scattered electron imaging.

Figure 11, center: A view of the front of the FSEI holder.

Figure 12, right: A view of the back of the FSEI holder, which faces the secondary electron detector. 


\section{from experience... learn from the experts.}

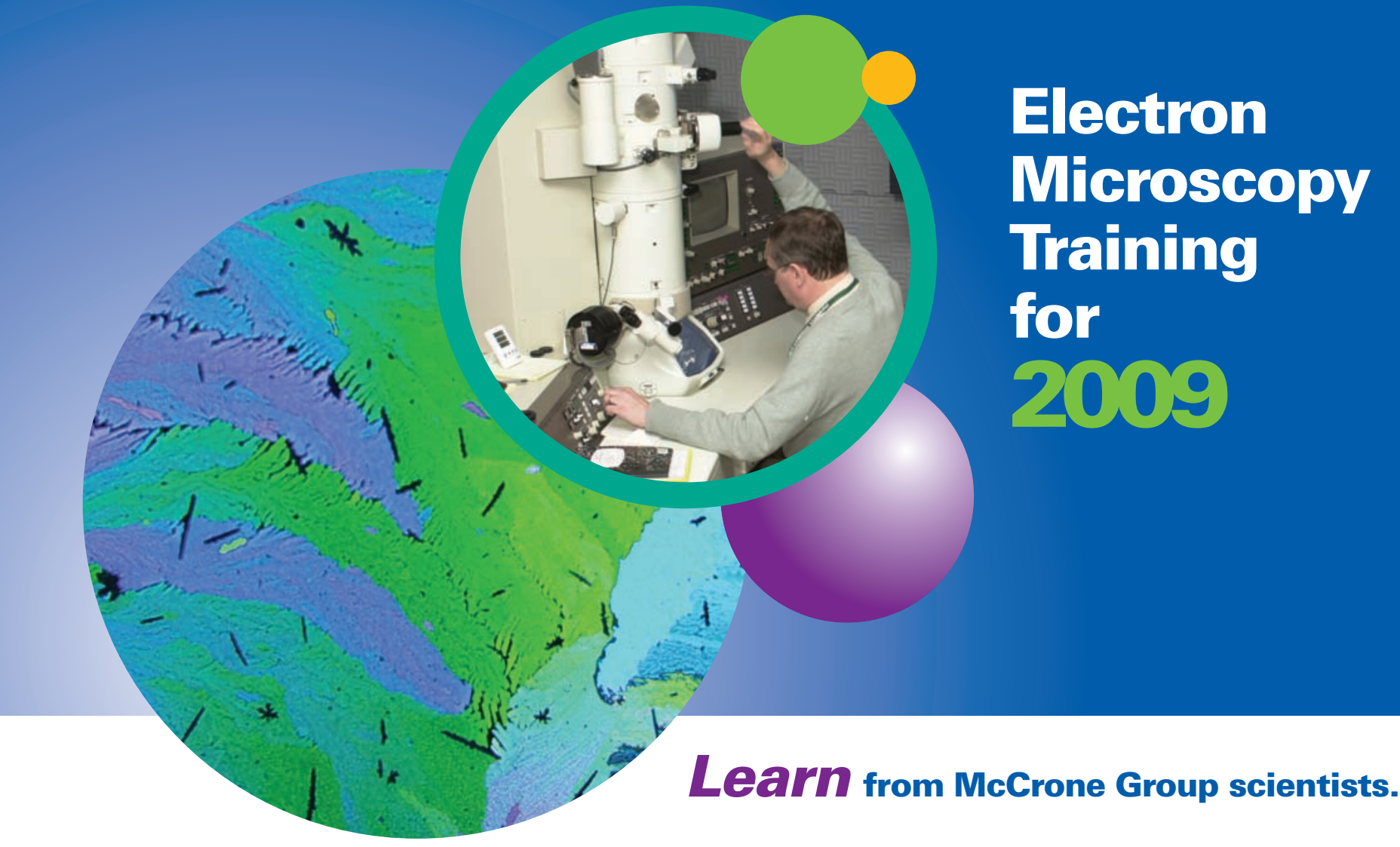

\section{COM200}

\section{Scanning Electron Microscopy}

Course provides in-depth lectures, demonstrations, and hands-on participation on how to setup and operate SEM and EDS instruments, including low-vacuum and field-emission models. Using multiple SEM and EMA instruments, students can study their own samples or image and analyze samples provided by our staff.

College of Microscopy SEM instructors have over 90 years of combined experience using SEM/EDS/WDS, and are members of the McCrone Associates analytical staff.

\section{Instructors:}

Kent L. Rhodes, Ph.D.; Joe M. Rebstock;

Wayne D. Niemeyer; Craig S. Schwandt, Ph.D.

Dates:

March 16-20, 2009

and October 5-9, 2009

\section{COM210}

\section{Advanced Scanning Electron Microscope Based Tools for Microanalysis}

Course concentrates on X-ray spectrometry, both energy dispersive (EDS) and wavelength dispersive (WDS), especially as it pertains to chemical or spectral mapping. The course also covers electron backscattered diffraction analysis, and touches on methods such as Monte Carlo modeling, cathodoluminescence, and sample preparation transfer.

Learn from a McCrone scientist with over 18 years experience.

Instructor:

Craig S. Schwandt, Ph.D.

Date:

February 9-13, 2009

\section{COM250 \\ Transmission Electron Microscopy}

Course provides practical, hands-on learning for new and experienced operators, utilizing lectures, demonstrations, direct student participation, and the use of JEOL JEM-3010 300 kV, LaB6 TEM. New TEM operators learn skills necessary to align a TEM, and select parameters for acquisition of images, EDS spectra, and electron diffraction patterns. Students with prior experience learn how to better utilize the multiple analytical capabilities of the TEM for real-world materials characterization.

Learn from instructors who have over 50 years of combined experience.

\section{Instructors:}

Alan Nicholls, Ph.D. and Elaine F. Schumacher.

Dates:

March 24-26, 2009

and October 27-29, 2009 

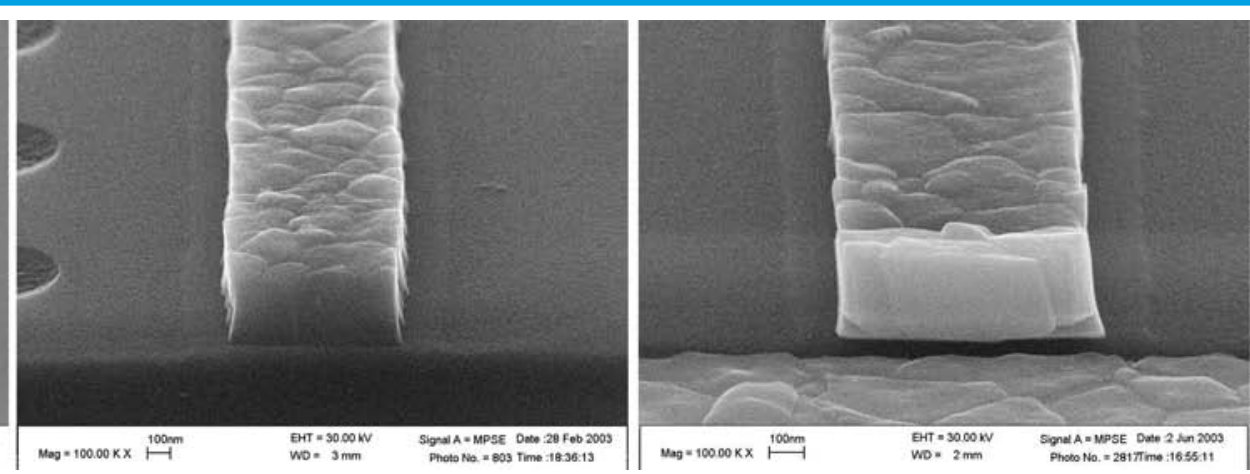

Figure 13, left: $700 \mathrm{~nm}$ poly lines on silicon, uncoated, $30 \mathrm{keV}$ beam energy, 70 degrees tilt, secondary electron imaging.

Figure 14, center: $700 \mathrm{~nm}$ poly lines on silicon, uncoated, $30 \mathrm{keV}$ beam energy, 70 degrees tilt, forward scattered electron imaging

Figure 15, right: $700 \mathrm{~nm}$ poly lines on silicon, uncoated, $30 \mathrm{keV}$ beam energy, 70 degrees tilt, forward scattered electron imaging. Dynamic Focus was

used to keep the entire sample in focus despite the high tilt angle.

for many types of metrology. If structures such as sub-0.1 micron poly lines are oriented orthogonal to the tilt axis, the line width and edge profile can be measured with very high resolution. Since the forward-scattered high energy beam is relatively unaffected by sample charging, it is also possible to image insulating materials like photo-resist or spin-on-glass at high beam voltage.

\section{Procedure}

The sample is mounted on a highly tilted surface near the top of the holder and held in place by metal clips. The sample surface is pointed away from the chamber secondary electron detector so that only the forward scattered electrons contribute to the image. Since most SEMs have their best resolution at very short working distance, the feature of interest should be mounted near the top of the holder. The maximum sample size for this holder design is about $1 \mathrm{~cm}$ by $1 \mathrm{~cm}$, although larger holders can be built. To use the sample holder in FSEI mode the stage tilt must be zero and the stage must be rotated so that the back of the holder points towards the chamber secondary electron detector. The sample cannot be re-oriented except by removing it from the vacuum chamber and remounting it.

\section{Polysilicon gate metrology}

0.7 micron polysilicon gate samples were prepared from a finished loose die by plasma etching to remove the passivation, followed by an HF dip to remove the metal and dielectric layers. A normal SEM image was first taken with the die face pointed towards the secondary electron detector (SED). The holder was then rotated $180^{\circ}$ so that the die surface was pointed away from the SED, and the only signal reaching the detector was from secondary electrons produced by the forward scattered electrons striking the gold reflectors.

Images taken using high beam voltage on uncoated samples typically have very poor contrast and little surface detail as seen in Figure 13 . However, when exactly the same beam conditions $(30 \mathrm{keV}, 4$ $\mathrm{mm}$ working distance, 30 micron aperture, $70^{\circ}$ tilt) were used in FSEI mode, the resulting image was far superior, see Figure 14. The image contrast and surface detail are both greatly improved. Note that this is an uncoated sample imaged at $30 \mathrm{keV}$. In many cases coating a sample is undesirable since the coating may obscure fine detail or interfere with microanalysis by energy dispersive x-ray or scanning Auger spectroscopy. Therefore, FSEI is particularly useful as a very high-resolution technique that does not require sputter coating the sample.

\section{Dynamic focus}

One disadvantage of using a high tilt angle is the difficulty in keeping the entire sample in focus due to limitations in the depth of field, particularly at the short working distances required for optimum resolution. Thus in Figure 14 the top part of the image lacks the sharp focus seen in the bottom of the image. However, many electron microscopes have a feature called dynamic focus that allows the electron beam focus to be automatically adjusted as the beam rasters down the image. This works quite well if the surface is flat as in Figure 15. Most of the FSEI images in this article were acquired using dynamic focus. The resolution of this image was measured to be about 1 to $2 \mathrm{~nm}$.

\section{Photoresist metrology}

Photo-resist is a highly insulating material that is usually imaged in the SEM with either a metal sputter coating or at low beam voltage $(\sim 1 \mathrm{keV})$. However, since the forward scattered low-loss electrons with close to $30 \mathrm{keV}$ energy are much less sensitive to sample charging than are the low energy secondary electrons with less than $50 \mathrm{eV}$ energy, it is possible to image uncoated resist with a $30 \mathrm{keV}$ beam using the forward scattering technique.

A photo-resist layer 1 micron thick on silicon dioxide was pat-
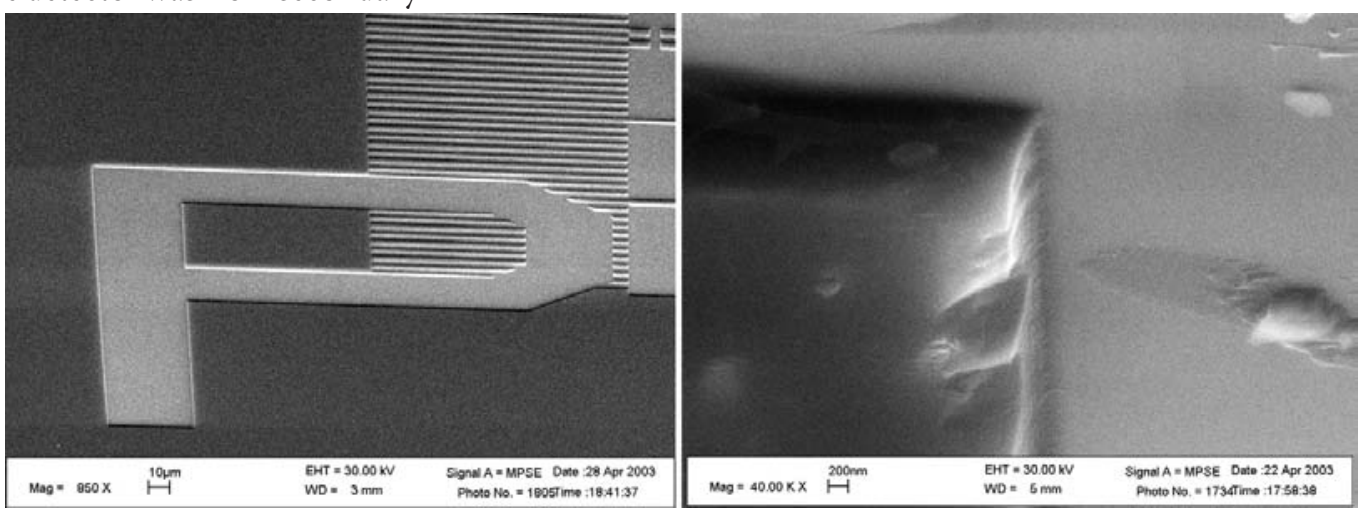

Figure 16, left: Photo-resist on silicon dioxide, uncoated, $30 \mathrm{keV}$ beam energy, 70 degrees tilt, forward scattered electron imaging.

Figure 17, right: Photo-resist on silicon dioxide, uncoated, $30 \mathrm{keV}$ beam energy, 70 degrees tilt, forward scattered electron imaging. 


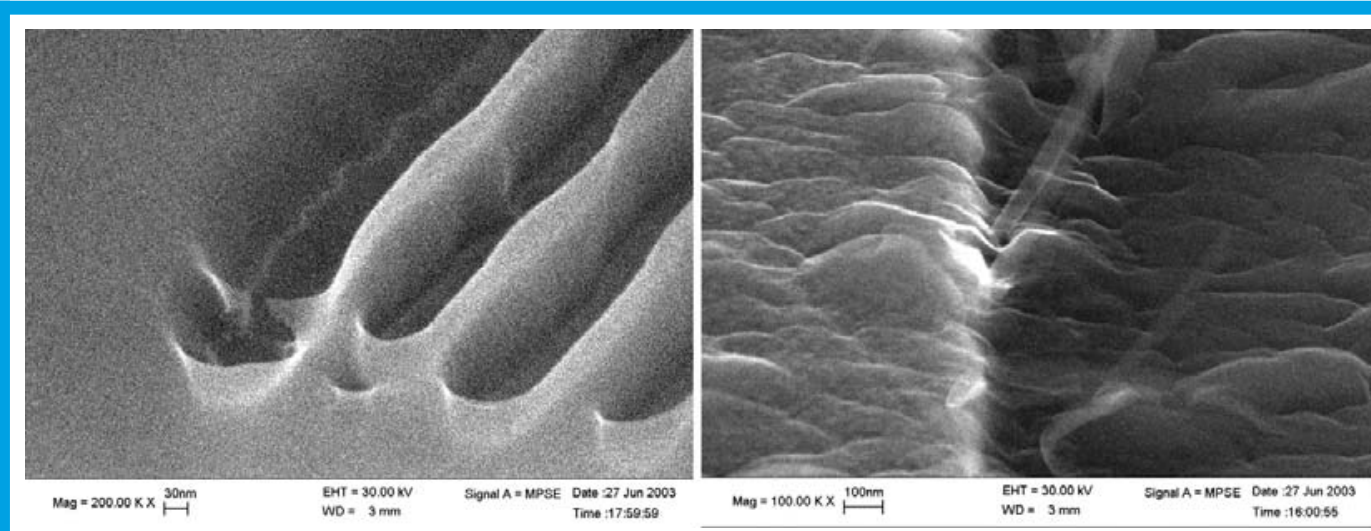

Figure 18: PMMA e-beam resist on silicon dioxide, uncoated, $30 \mathrm{keV}$ beam energy, 70 degrees tilt, forward scattered electron imaging.

Figure 19: Pentacene thin film on gold (left) and oxide (right), uncoated, $30 \mathrm{keV}$ beam energy, 70 degrees tilt, forward scattered electron imaging.

terned and developed with a test structure, and then imaged with a $30 \mathrm{keV}$ electron beam using the forward scattered technique, see Figs. 16 and 17. No charging or sample damage was observed. Since the resist has a lower density than silicon or metals the incident beam should tend to penetrate deeper into the sample before being scattered towards the detector. Thus, it was expected that the spatial resolution and surface sensitivity would be reduced when imaging resist compared to imaging other semiconductor materials. However, Figure 17 at $40,000 \times$ is amazingly sharp and surface sensitive given the high beam voltage and lack of any sputter coating on the resist. To an electron microscopist who works with photoresist, this is a shocking image. The physical mechanism for this result is not yet fully understood.

A PMMA (poly methyl methacrylate acid) e-beam resist layer $250 \mathrm{~nm}$ thick was patterned by electron beam lithography with 50 $\mathrm{nm}$ lines, see Figure 18. Although the contrast in this $200,000 \times$ image is marginal, the $50 \mathrm{~nm}$ lines are clearly visible, which is a remarkable result since very fine resist structures such as this are almost always sputter coated before imaging. FSEI imaging of uncoated resist lines below $100 \mathrm{~nm}$ represents a new metrology capability.

Figure 19 is from an actual failure analysis problem, an investigation of the grain structure of the organic semiconductor pentacene. In Figure 19, a $50 \mathrm{~nm}$ thick pentacene film covers a gold electrode on the left side of the image, and thermal oxide on the right side of the image. The grain size is reduced on the gold electrode, which affects device performance. It was also noted that $50 \mathrm{~nm}$ diameter extrusions of pentacene occur at the gold / oxide interface.

\section{Image correction}

The use of high tilt angle foreshortens the image resulting in image distortion. However, it is easy to correct this distortion by resizing the vertical scale of the image with any standard graphics program. Some scanning electron microscopes have this feature labeled as "tilt correction." Note that image correction works well for very flat samples, but any features that protrude out of the flat plane of the sample will be severely distorted by this procedure.

\section{Beam damage}

High energy electron beams will sometimes damage sensitive materials such as polymers. However, in taking several dozen very high magnification images on polymer films, damage from melting, vaporization, or electrostatic discharge was never observed, despite using slow scan at 200,000x and focusing and stigmating at $800,000 \times$ with $1 / 3$ partial field. The lack of damage is somewhat surprising, but may be explained by the high tilt angle which distributes the deposited energy across a large surface area. In addition, the energy absorption per path length falls as the beam energy increases so the actual energy deposition at the point of impact is less for higher beam energy.

\section{Discussion}

What physical effects produce the contrast in the FSEI images? In FSEI, electrons are scattered towards the target when they undergo collisions with atoms in the sample. In this aspect, the technique is more similar to TEM (transmission electron microscopy) than to SEM. One might almost be tempted to refer to forward scattered imaging as "glancing angle TEM" especially since the magnification achieved by this technique is most often associated with TEM imaging.

Despite the far simpler hardware, FSEI images are in many cases superior to the low-loss electron images published in the literature. This confirms that collection of a small solid angle of the forward scattered beam is more effective at selecting the high-resolution and surface-sensitive electrons than selecting out the low-loss electrons with an electron energy filter.

\section{Conclusion}

Techniques like STEM-in-SEM and FSEI allow us to "break the resolution barrier" in the SEM. Self-contained sample-holder/ detectors enable these techniques to be performed in an ordinary unmodified SEM. Approximately 1 to $2 \mathrm{~nm}$ resolution images can be obtained on thin sections, uncoated polysilicon gates, and photoresist. These are promising techniques for semiconductor sub-100 $\mathrm{nm}$ metrology and failure analysis. These techniques will probably have applications in biological SEM imaging.

\section{References}

1. L. Tsung, A. Anciso, R. Turner, T. Dixon, and N. Holloway, Proceedings of ISTFA 2001, p. 299-302 (2001).

2. W. E. Vanderlinde, Proceedings of ISTFA 2002 pp. 77-85 (2002).

3. E. Coyne, Proceedings of ISTFA 2002 pp. 93-99 (2002).

4. B. Tracy, Proceedings of ISTFA 2002, pp. 69-76 (2002).

5. W. E. Vanderlinde, Proceedings of ISTFA 2003 pp. 158-165 (2003).

6. Joseph I. Goldstein, Dale E. Newbury, Patrick Echlin, David C. Joy, A. D. Romig, Jr., Charles E. Lyman, Charles Fiori, and Eric Lifshin, Scanning Electron Microscopy and X-ray Microanalysis, A Textbookfor Biologists, Materials Scientists, and Geologists, $2^{\text {nd }}$ Edition, Plenum, New York, 1992, p. 219.

7. Private communication, Peter Gnauck, LEO Electron Microscopy GmbH.

8. Goldstein, et al., p. 89.

9. Goldstein, et al., p. 269.

10. O.C. Wells, Appl. Phys. Lett. 19 (7) p. 232-235 (1971).

11. O.C. Wells, Appl. Phys. Lett. 49 (13) p. 764-766 (1986).

12. O.C. Wells, F.K. LeGoues, and R.T. Hodgson, Appl. Phys. Lett. 56 (23) p. 2351 2353 (1990).

13. A.N. Broers, B.J. Panessa, and J.F. Gennaro, Proceedings of the Eighth Annual Scanning Electron Microscope Symposium (SEM/1975), IIT Research Institute, Chicago, p. 233-242 (1975) 